\title{
Response maxima in time-modulated turbulence: Direct Numerical Simulations
}

\author{
Arkadiusz K. $\left.\operatorname{Kuczaj}^{1}{ }^{*}\right)$, Bernard J. Geurts ${ }^{1,2}$ and Detlef $\left.\operatorname{Lohse}^{3}{ }^{(* *}\right)$ \\ 1 Department of Applied Mathematics - University of Twente, \\ P.O. Box 217, 7500 AE Enschede, the Netherlands \\ 2 Fluid Dynamics Laboratory - Eindhoven University of Technology, \\ P.O. Box 513, 5300 MB Eindhoven, the Netherlands \\ 3 Department of Applied Physics - University of Twente, \\ P.O. Box 217, 7500 AE Enschede, the Netherlands
}

PACS. 47.27.Rc - Turbulence control.

PACS. 47.27.Eq - Turbulence simulation and modeling.

PACS. 47.27.Gs - Isotropic turbulence; homogeneous turbulence.

\begin{abstract}
The response of turbulent flow to time-modulated forcing is studied by direct numerical simulations of the Navier-Stokes equations. The large-scale forcing is modulated via periodic energy input variations at frequency $\omega$. The response is maximal for frequencies in the range of the inverse of the large eddy turnover time, confirming the mean-field predictions of von der Heydt, Grossmann and Lohse (Phys. Rev. E 67, 046308 (2003)). In accordance with the theory the response maximum shows only a small dependence on the Reynolds number and is also quite insensitive to the particular flow-quantity that is monitored, e.g., kinetic energy, dissipation-rate, or Taylor-Reynolds number. At sufficiently high frequencies the amplitude of the kinetic energy response decreases as $1 / \omega$. For frequencies beyond the range of maximal response, a significant change in phase-shift relative to the time-modulated forcing is observed.
\end{abstract}

Introduction. - Recently, response maxima in time modulated turbulence have been predicted within a mean field theory of turbulence [1]. Subsequently, such response maxima were found [2] in numerical simulations of simplified dynamical turbulence models such as the GOY model [3-5] or the reduced wave vector approximation (REWA) [6-9]. However, these response maxima computed in [2] were not pronounced at all, due to the approximate treatment of the small scales in either of these approaches. Indications of response maxima resulting from time-modulated forcing have subsequently also been seen in experiment [10]. The experimental observations were done by introducing a time-dependent swirl to fluid in a closed container and monitoring the energy-dissipation-rate. The selected set-up did not allow to identify possible flow-structuring under resonance conditions, nor to conclusively distinguish such resonance phenomena from flow-organization associated with the size of the container.

\footnotetext{
$\left({ }^{*}\right)$ e-mail: a.k.kuczaj@utwente.nl

$\left({ }^{*}\right)$ e-mail: d.lohse@utwente.nl
}

(c) EDP Sciences 
The purpose of this paper is to complement these theoretical, numerical, and experimental observations by direct numerical simulations (DNS) of turbulence, subject to time-modulated large-scale forcing. In a turbulent flow whose large-scale forcing is periodically modulated in time, all typical flow-properties develop a complex time-dependence. However, averaging such turbulent time-dependence, conditioned on the phase of the periodic modulation, yields a clear and much simpler periodic pattern [2]. The dependence of the conditionally averaged response on the frequency of the modulation may be quantified by monitoring changes in flow-properties such as total energy, dissipation-rate, or Taylor-Reynolds number. In case of a fast modulation with a frequency $\omega \gg \omega_{L}$, where $\omega_{L}$ is the inverse large eddy turnover time, only a modest effect on the flow is expected, or none at all. Likewise, if $\omega \ll \omega_{L}$ the modulation is quasistationary and the flow may be expected to closely resemble the corresponding unmodulated case. In between these extremes a more pronounced response may develop, which is the subject of this investigation.

The DNS approach allows to investigate in detail the response of turbulent flow-properties to periodic modulation of the forcing. In particular, we present an extensive parameterstudy involving a large range of modulation frequencies for two different Reynolds numbers, and establish response maxima in a variety of flow-properties. The response is found to be significantly increased at modulation frequencies on the order of the inverse of the eddyturnover time. Near resonance, the 'activity' of the turbulent flow is found to be considerably higher than in the unmodulated case. At high frequencies $\omega$ the amplitude of the modulationspecific response of the kinetic energy is found to uniformly decrease to zero as $\omega^{-1}$. This type of external control of turbulence may offer new opportunities with relevance to technological applications.

The organization of this paper is as follows. We first introduce the computational flowmodel in more detail. Subsequently, an overview of the ensemble averaging procedure and data extraction is given. Then the main result, the response of various flow properties to timemodulated forcing, is presented. The paper ends with a summary and conclusions.

Computational flow-model. - The full Navier-Stokes equations for incompressible flow are numerically solved in a periodic flow-domain with a pseudo-spectral code. In spectral space, the Navier-Stokes equations read

$$
\left[\frac{\partial}{\partial t}+\nu|\mathbf{k}|^{2}\right] u_{\alpha}(\mathbf{k}, t)=M_{\alpha \beta \gamma}(\mathbf{k}) \sum_{\mathbf{p}+\mathbf{q}=\mathbf{k}} u_{\beta}(\mathbf{p}, t) u_{\gamma}(\mathbf{q}, t)+F_{\alpha}(\mathbf{k}, t)
$$

with $M_{\alpha \beta \gamma}(\mathbf{k})=\frac{1}{2 \imath}\left(k_{\beta} D_{\alpha \gamma}(\mathbf{k})+k_{\gamma} D_{\alpha \beta}(\mathbf{k})\right)$, with $D_{\alpha \beta}(\mathbf{k})=\delta_{\alpha \beta}-k_{\alpha} k_{\beta} /|\mathbf{k}|^{2}$. Here, $\nu$ is the kinematic viscosity, $u_{\alpha}(\mathbf{k}, t)$ is the Fourier-coefficient of the velocity field at wave vector $\mathbf{k}$ and time $t$ and $F_{\alpha}$ is the time-modulated forcing.

First, we recall that traditional agitation of the large-scale structures in a turbulent flow may be achieved by introducing a forcing term restricted to wave vectors with $|\mathbf{k}| \leq k_{F}$, i.e., identifying a forcing-range through the upper-limit $k_{F}$. Specifically, we force the turbulence similarly as in $[7,11]$,

$$
f_{\alpha}(\mathbf{k}, t)=\frac{\varepsilon_{w}}{N_{F}} \frac{u_{\alpha}(\mathbf{k}, t)}{|\mathbf{u}(\mathbf{k}, t)|^{2}} \quad ; \quad|\mathbf{k}|<k_{F}
$$

where $\varepsilon_{w}$ is the constant energy injection rate and $N_{F}=N_{F}\left(k_{F}\right)$ is the total number of forced modes. For convenience, the wave vectors are grouped in spherical shells with the $n$-th shell containing all modes such that $(n-1 / 2)<|\mathbf{k}| \leq(n+1 / 2)$. We applied large-scale forcing either in the first shell at $n=1$ (i.e., $k_{F}=3 / 2$ which implies $N_{F}=18$, the case considered 
in [2]) or in the first two shells (i.e., $k_{F}=5 / 2$ which implies $N_{F}=80$ ). The second step in specifying the forcing $F_{\alpha}$ introduces the periodic time modulation

$$
F_{\alpha}(\mathbf{k}, t)=f_{\alpha}(\mathbf{k}, t)\left(1+A_{F} \sin (\omega t)\right),
$$

where $A_{F}$ is the amplitude of modulation and $\omega$ its angular frequency. The modulated forcing corresponds to a total energy input rate which oscillates around $\varepsilon_{w}$ with amplitude $A_{F}$,

$$
T_{F}(\omega, t)=\sum_{\mathbf{k}} u_{\alpha}^{*}(\mathbf{k}, t) F_{\alpha}(\mathbf{k}, t)=\varepsilon_{w}\left(1+A_{F} \sin (\omega t)\right) .
$$

The lengths and time scales of the numerical simulation are chosen by picking $L=1$ for the box-size in physical space and $\varepsilon_{w}=0.15$ for the energy injection rate. The Reynolds number is then determined by the dimensionless viscosity $\nu$. Choosing $\nu^{-1}=1060.7$ and $\nu^{-1}=4242.6$ result in respective approximate Taylor-Reynolds numbers $R_{\lambda} \cong 50$ and $R_{\lambda} \cong 100$. We use these two cases as references denoted by $R_{50}$ and $R_{100}$. The spatial resolution needed may be estimated by requiring $k_{\max } \eta>1$ [12] with $\eta$ the Kolmogorov dissipation scale and $k_{\max }$ the highest wavenumber included in the spatial discretization. For $R_{50}$ case a resolution of at least $N^{3}=64^{3}$ computational points is required while for $R_{100}$ a higher resolution of $192^{3}$ points is necessary. The latter poses a strong computational challenge in view of the extensive ensemble averaging and large number of modulation frequencies. However, it was found that many large scale quantities, such as the total energy, do not depend too sensitively on resolution. As an example, a resolution of $64^{3}$ points corresponds to $k_{\max } \eta \approx 0.4$ for the $R_{100}$ case. Still, this resolution is quite adequate for studying the response of total energy. This was verified by repeating the analysis at a selection of modulation frequencies with resolutions $128^{3}$ and $192^{3}$. The predictions of quantities that rely more on small scales, such as the dissipation-rate, contain a higher numerical uncertainty for $R_{100}$ case and $64^{3}$ computational points, but still allow a clear interpretation of the main turbulence response. This was separately assessed using the higher resolution data at selected characteristic frequencies.

The direct numerical simulation for the unmodulated case starts from an initial condition that was generated on the basis of the Pao spectrum [13]. We adopt exactly the same initial conditions as in [14] which allow a separate validation of the simulations. Explicit second order compact-storage Runge-Kutta time-stepping [15] with fully de-aliased pseudo-spectral discretization is used. The unmodulated turbulent flows provide the point of reference for investigating the effect of modulated forcing, to which we turn next.

Averaging procedure and simulation setting. - In order to analyze the response to a timemodulated forcing, the precise extraction of the amplitude and phase of the conditionally averaged variations is a key issue. Two steps can be distinguished, i.e., the computation of the conditionally averaged signal itself and the subsequent determination of amplitude and phase characteristics of this signal, see Figure 1. These steps are discussed and illustrated next.

We adopt ensemble averaging to determine the conditionally averaged signal $S(\omega, t)$, where $S(\omega, t)$ is the total energy $E(\omega, t)$, the Taylor-Reynolds number $R_{\lambda}(\omega, t)$ or the energy dissipation rate $\varepsilon(\omega, t)$. Ensemble averaging requires a sufficiently large sample of statistically independent signals $\left\{S_{j}(\omega, t)\right\}$ to be generated. The computational approach is summarized in Figure 1(a) and involves two main steps. Firstly, we compute the unmodulated flow and store $N_{r}$ realizations of the turbulent solution corresponding to $t>10$. The latter condition allows transients related to the initial condition to become negligible. The time-separation between these snapshots is larger than two eddy-turnover times. Subsequently, each of these 

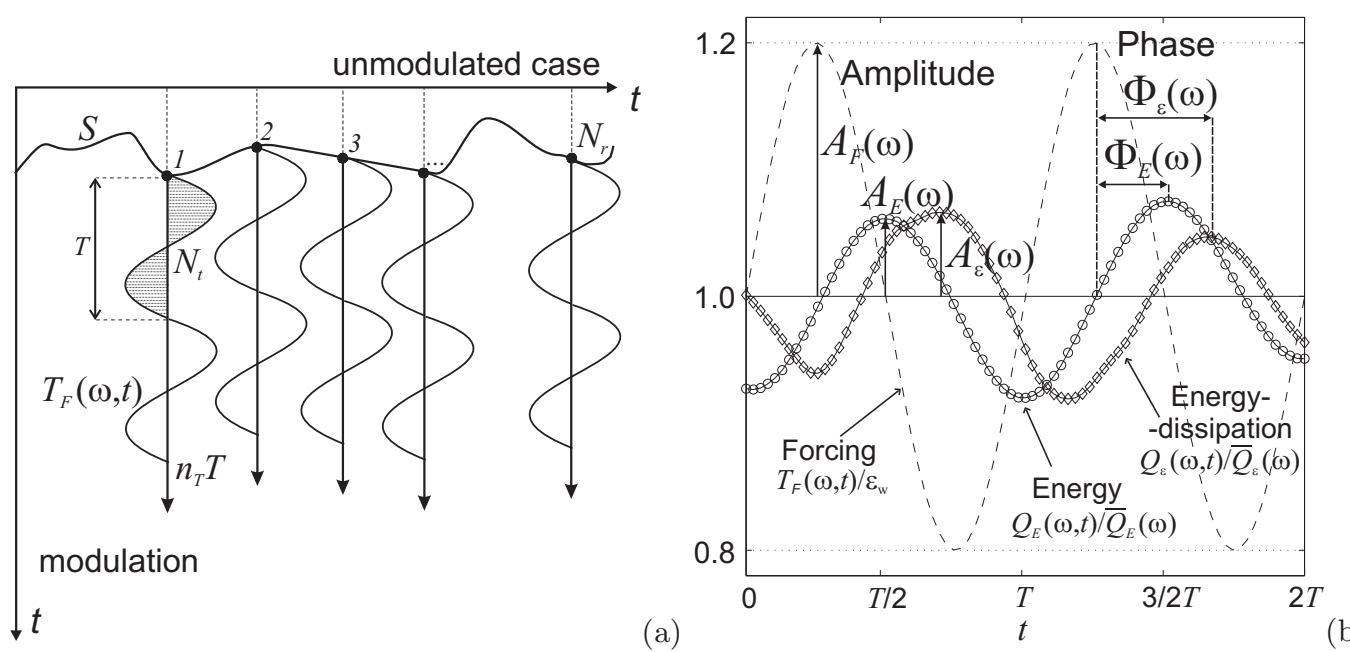

Fig. 1 - Procedure of data generation to compute the conditional average (a) and the amplitudes $A_{F}(\omega), A_{E}(\omega), A_{\varepsilon}(\omega)$ and phase-shifts $\Phi_{F}(\omega) \equiv 0, \Phi_{E}(\omega), \Phi_{\varepsilon}(\omega)$ of the forcing $T_{F}(\omega, t)$ (dashed line), the energy $Q_{E}(\omega, t)$ (labeled $\left.\circ\right)$, and the energy-dissipation-rate $Q_{\varepsilon}(\omega, t)$ (labeled $\left.\diamond\right)$ normalized by their respective means $\bar{T}_{F}=\varepsilon_{w}, \bar{Q}_{E}(\omega)$, and $\bar{Q}_{\varepsilon}(\omega)$ obtained from simulations at the modulation frequency $\omega=0.8 \pi(b)$.

$N_{r}$ realizations was taken as the initial condition for a simulation with time-modulated forcing at a particular frequency $\omega$. This provides $N_{r}$ sample signals which need to be averaged to obtain the conditionally averaged signal $S(\omega, t)$. Repeating this procedure for a range of frequencies yields the total response characteristics. Given the conditionally averaged response signal $S(\omega, t)$, there are various ways in which amplitude and phase information can be extracted. In [10] the signal $S(\omega, t)$ was first averaged over time to yield $\bar{S}(\omega)$. Subsequently, the normalized variation defined as $Q_{S}^{(a)}(\omega, t)=S(\omega, t) / \bar{S}(\omega)$ was studied using the Fourier-transform $(\mathcal{F})$ in which time $t$ is transformed into frequency $f$. Correspondingly, the power amplitude spectrum $\widehat{Q}_{S}^{(a)}(\omega, f)=\mathcal{F}\left(Q_{S}^{(a)}(\omega, t)-1\right)$ can be obtained which assumes a maximum value $A_{S}(\omega)=\left.\max \left\{\left|\widehat{Q}_{S}^{(a)}(\omega, f)\right|\right\}\right|_{f=f_{A}(\omega)}$, as denoted in Figure $\mathrm{W}$ b) for forcing $A_{F}(\omega)$, total energy $A_{E}(\omega)$, and energy-dissipation-rate $A_{\varepsilon}(\omega)$. The maximum $A_{S}(\omega)$ as the amplitude at dominant frequency can be used to quantify the response as function of the modulation frequency $\omega$. This approach is accurate if Fourier-transform is applied to an integer number of modulation periods. The method used in [2] is based on a fitting procedure in which it is assumed that $S(\omega, t) \approx \bar{S}+A_{S} \sin \left(\omega t+\Phi_{S}\right)$. The dependence of the parameters $\left\{\bar{S}, A_{S}, \Phi_{S}\right\}$ on $\omega$ may be obtained from a least squares procedure. This evaluation method assumes that the conditionally averaged signal has the same frequency as the forcing.

At modest ensemble-size $N_{r}$ it is beneficial to explicitly incorporate variations in the unmodulated reference signal to improve the data-evaluation. This motivates an alternative method in which we determine $N_{r}$ sample signals $\left\{S_{j}(\omega, t)\right\}$ corresponding to the modulated case, as well as $N_{r}$ unmodulated signals $\left\{s_{j}(t)\right\}$ that start from the same set of initial conditions. This allows to generate different 'normalized' signals such as $Q_{S}^{(b)}(\omega, t)=\sum_{j} S_{j} / \sum_{j} s_{j}$ or $Q_{S}^{(c)}(\omega, t)=\sum_{j} S_{j} / s_{j} / N_{r}$. These normalized signals provide estimates that compensate to some degree for the relatively small number of samples or for an unknown mean component 
but have the drawback that they cannot be applied in the context of a physical experiment. Additionally, we divided these signals by its means (time-averages) and removed the constant component corresponding to the zero-frequency response. Application of the Fouriertransform, $\widehat{Q}_{S}^{(b)}=\mathcal{F}\left(Q_{S}^{(b)} / \bar{Q}_{S}^{(b)}-1\right)$ and $\widehat{Q}_{S}^{(c)}=\mathcal{F}\left(Q_{S}^{(c)} / \bar{Q}_{S}^{(c)}-1\right)$, provides direct access to amplitude and phase information. Each of these methods identified above yields the same general impression of response maxima in time-modulated turbulence. Differences arise only on a more detailed level of the processed data but these do not obscure the interpretation of the main features of the response. Therefore we only present results extracted from the normalized signal $Q_{S} / \bar{Q}_{S} \equiv Q_{S}^{(c)} / \bar{Q}_{S}^{(c)}$ in what follows, unless explicitly stated otherwise. The simulations were performed in the frequency range $\pi / 5 \leq \omega \leq 80 \pi$ with time-modulated forcing at an amplitude $A_{F}=1 / 5$. Referring to Figure 1 for each of the $N_{r}$ unmodulated initial conditions, $n_{T}=4$ periods of the modulated forcing were simulated, i.e., each sample signal was computed for $n_{T} T$ time-units with modulation-period $T=2 \pi / \omega$. Since an explicit time-stepping method was adopted, the cases at low $\omega$ add particularly to the total computational cost. The number of realizations required in the ensemble was investigated separately. Results for several modulation frequencies were compared at $N_{r}=10,30$ and 50; it was found that 30 independent samples provide adequate statistical convergence for our purposes. We stored $N_{t}=40$ points per modulation period and present results obtained by evaluating the last two recorded periods, i.e., $2 T \leq t \leq 4 T$. Comparison with results obtained by evaluating data on $0 \leq t \leq 4 T$ yielded only minor differences. Finally, the phase $\Phi_{S}(\omega)$ between the forcing and the response can be computed from the Fourier-transformed data as well. At the dominant frequency $f_{A}$ of the transformed signal $\left.\widehat{Q}_{S}(\omega, f)=\mathcal{F}\left(Q_{S}(\omega, t)\right) / \bar{Q}_{S}(\omega, t)-1\right)$, the phase becomes $\Phi_{S}(\omega)=\arctan \left(\operatorname{Im}\left(\widehat{Q}_{S}\left(\omega, f_{A}\right)\right) / \operatorname{Re}\left(\widehat{Q}_{S}\left(\omega, f_{A}\right)\right)\right)$.
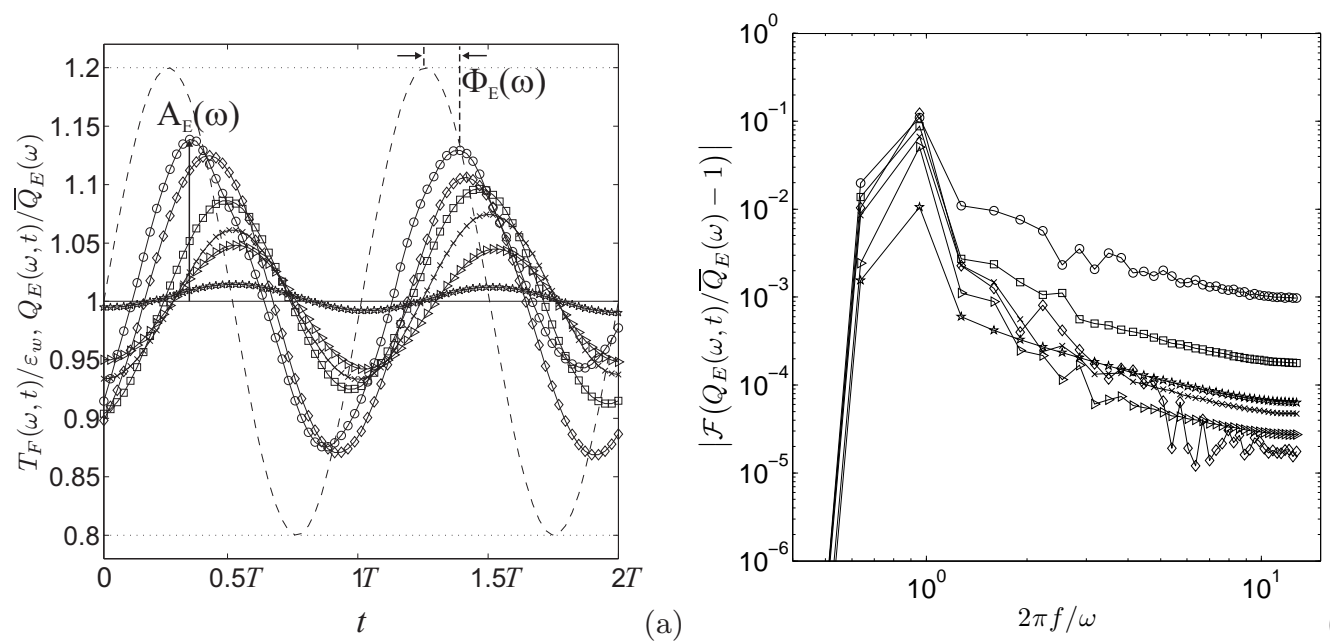

Fig. 2 - The response $Q_{E}(\omega, t) / \bar{Q}_{E}(\omega)$ for the $R_{50}$ case recorded at different modulation frequencies $\omega$ is shown in (a) together with the modulation of the forcing $T_{F}(\omega, t) / \varepsilon_{w}$ (dashed). The corresponding power spectra of the Fourier-transform as function of the transformed frequency $f$ are collected in (b). Modulation frequencies $\omega /(2 \pi)=0.1,0.2,0.3,0.4,0.5,2.0$ are included and labeled by $\circ, \diamond, \square, \times, \triangleright$, and $\star$, respectively. 
Modulated turbulence. - In Figure2(a) the conditionally averaged signal $Q_{E}(\omega, t) / \bar{Q}_{E}(\omega)$ based on total energy is shown at a number of modulation frequencies. The conditionally averaged response has a clear oscillatory behavior. The Fourier-transform of the data from Figure 2 (a) is shown in Figure 2 b) and displays a dominant maximum corresponding to the forcing frequency $f_{A}=\omega /(2 \pi)$. This observation confirms that the least-squares fitting procedure adopted in $[2]$ is justified.
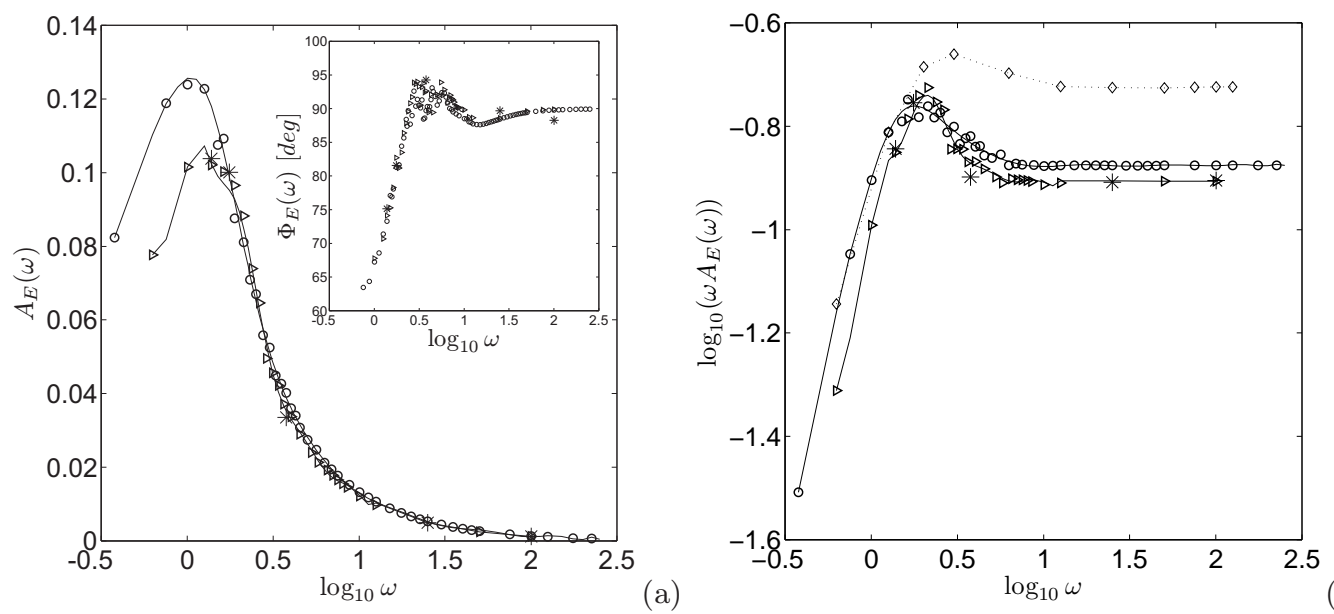

Fig. 3 - (a) Amplitude of the energy response $A_{E}(\omega)$ and (b) compensated energy response $\omega A_{E}(\omega)$ obtained for the $R_{50}$ (labeled $\circ$ ) and the $R_{100}$ (labeled $\triangleright$ ) cases. Verification at selected frequencies for resolution $128^{3}$ and $R_{100}$ case (labeled $\star$ ). Results for forcing in two first shells $\left(k_{F} \leq 5 / 2\right)$ and the $R_{50}$ case (labeled $\left.\diamond\right)$. The inset in (a) shows the phase-shift $\Phi_{E}(\omega)$ between the energy response and the forcing modulation.

We now focus on the amplitude of the total energy response as function of the modulation frequency $\omega$. The amplitude $A_{E}(\omega)$ computed as maximum of the Fourier-transformed normalized signal for each modulation frequency is shown in Figure 3(a). The maximum response appears at $\omega_{\max } \approx 1.5$, in accordance with the expectation $[1,2]$ that it should be close to the inverse large eddy turnover time. In addition, the location of the maximum is not very sensitive to $R_{\lambda}$, reflecting that the response maximum is mainly associated with the large-scale features in the flow. At high modulation frequencies $\omega>\omega_{\max }$ the decay of $A_{E}$ is proportional to $\omega^{-1}$, which becomes particularly visible in the compensated response $\omega A_{E}(\omega)$, Figure 3(b). At very low modulation frequencies $\omega<\omega_{\max }$ a plateau in $A_{E}(\omega)$ must of course develop $[1,2]$, as the turbulence then completely follows the forcing. Our simulations do not achieve small enough $\omega$ to observe a pronounced plateau.

The maximum of $\omega A_{E}(\omega)$ is about $35 \%$ higher as compared to the value at high $\omega$. This is as expected lower than predicted by the mean-field theory described in [1] as the fluctuations slightly smear out the mean-field maximum, but it is much more pronounced compared to results based on the GOY or REWA simulations [2]. The reason is that, although the appearance of the response maxima is a large-scale effect, the correct resolution of the smallscales is important for a proper quantitative representation of the effect, because the small scale resolution affects the energy flux downscale. We also calculated the response curves for the Taylor-Reynolds number; the results are quite similar.

The phase-difference between the forcing modulation and the conditionally averaged total 

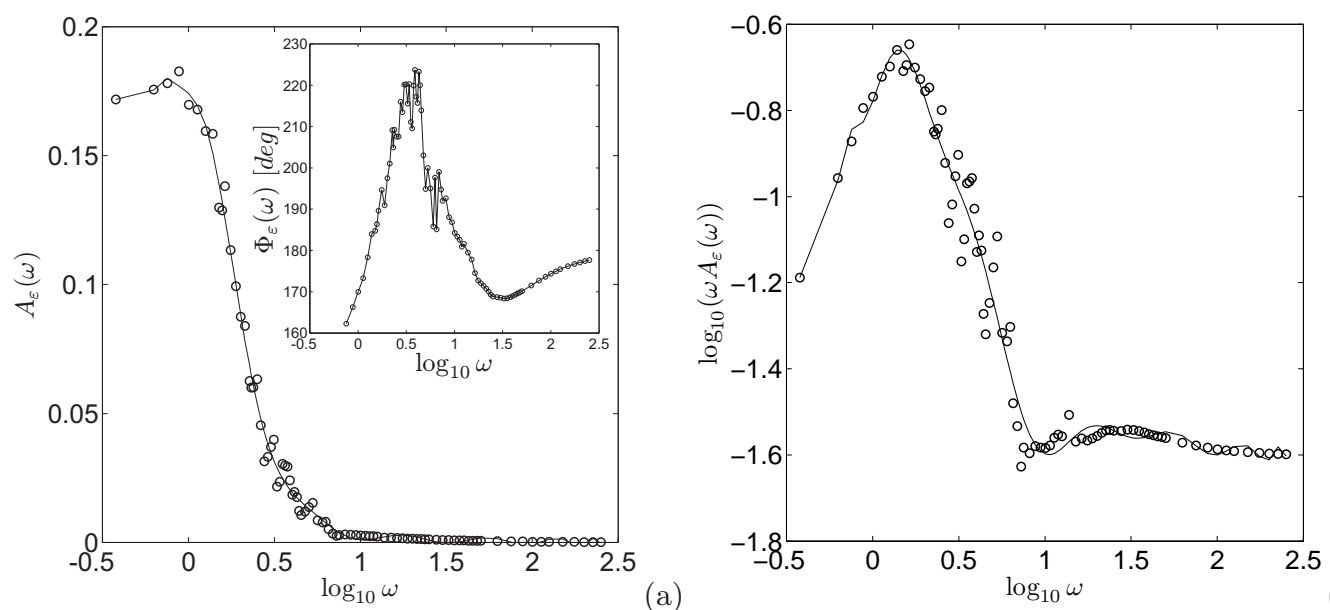

(a)

Fig. 4 - (a) Amplitude of the energy-dissipation-rate response $A_{\varepsilon}(\omega)$ and (b) compensated energydissipation-rate response $\omega A_{\varepsilon}(\omega)$ obtained for the $R_{50}$ case. The inset in (a) shows the phase-shift $\Phi_{\varepsilon}(\omega)$ between the energy-dissipation response and the forcing modulation.

energy response is shown in Figure 3(a) as inset. We observe a strong variation in this phase-difference for modulation frequencies near the most responsive modulation frequency. It appears that the maximum response as shown in Figure 3 occurs at a modulation frequency where also the variation in the phase-difference is largest. A strong phase shift was found similarly in windtunnel experiments in which a time-modulation is introduced via a periodic cycling of an upstream active grid. In these experiments the maximum response was found to shift to higher frequencies in case the characteristic length-scales of the forcing were reduced.

Can such a dependence on the type of forcing also be observed in our numerical simulations? To find out we force a higher wavenumber band of modes $\left(k_{F} \leq 5 / 2\right)$ instead of restricting us entirely to low wavenumber forcing. The result is seen in Figure 3 (b) indicated by diamonds. Indeed, for this type of forcing the response maximum is less pronounced. Further quantitative connections with physical experiments are currently being investigated.

The energy-dissipation-rate in the system is a quantity that is accessible to direct physical experimentation. In Figure 4 we show the energy-dissipation-rate amplitude $A_{\varepsilon}(\omega)$. We notice that at high modulation frequency $\omega$ the amplitude approaches zero, consistent with the expectation that the modulation of the forcing is not effective in this range. More importantly, the energy-dissipation-rate amplitude displays a strong response maximum at the level of $85 \%$ compared to the amplitude of modulation. The total mean energy-dissipation $T^{-1} \int_{0}^{T} \varepsilon(\omega, t) d t$ for each modulation frequency $\omega$ is almost constant. It differs from the energy input rate $\varepsilon_{w}=0.15$ at the level of $1 \%$ for most of the frequencies, reaching the maximum difference of $5 \%$ for the lowest simulated frequency, confirming good numerical convergence.

Summary and Conclusions. - The direct numerical simulation of the response of turbulence to time-modulated forcing confirms the existence of a response maximum. The simulation findings are in general agreement with predictions based on a mean-field theory [1]. The mean-field theory predicts the decrease of the response amplitude proportional to $\omega^{-1}$ as the modulation frequency is sufficiently large which was observed in the simulations as well. The response maxima in the total energy and the Taylor-Reynolds number occur at the forcing 
frequencies of the order of the inverse large eddy turnover time scale. The phase-difference between the modulation of the forcing and the conditionally averaged response displays a strong dependence on the modulation frequency as well. The modulation frequency at which the response maximum arises depends only weakly on the Reynolds number but shows a dependence on the scales included in the forcing as well as on the flow-property that is considered. In general, if the particular quantity of interest shows a stronger dependence on the smaller scales in a turbulent flow, then the response maximum arises at a somewhat higher frequency. These findings may be independently assessed in physical experiments, e.g., conducted in wind tunnels combined with the use of active grids cycled in a periodic sequence [16].

Stimulating discussions with Willem van de Water (Eindhoven University of Technology) are gratefully acknowledged. This work is part of the research program 'Turbulence and its role in energy conversion processes' of the Foundation for Fundamental Research of Matter (FOM), in the Netherlands, which is financially supported by the Netherlands Organization for Scientific Research (NWO). The authors wish to thank SARA Computing and Networking Services in Amsterdam for providing the computational resources.

\section{REFERENCES}

[1] A. von der Heydt, S. Grossmann, and D. Lohse, Phys. Rev. E 67, 046308 (2003).

[2] A. von der Heydt, S. Grossmann, and D. Lohse, Phys. Rev. E 68, 066302 (2003).

[3] L. Biferale, E. Calzavarini, F. Toschi, and R. Tripiccione, Europhys. Lett. 64, 461 (2003).

[4] T. Bohr, M. H. Jensen, G. Paladin, and A. Vulpiani, Dynamical Systems Approach to Turbulence (Cambridge University Press, Cambridge, 1998).

[5] L. Kadanoff, D. Lohse, J. Wang, and R. Benzi, Phys. Fluids 7, 617 (1995).

[6] J. Eggers and S. Grossmann, Phys. Fluids A 3, 1958 (1991).

[7] S. Grossmann and D. Lohse, Z. Phys. B 89, 11 (1992).

[8] S. Grossmann and D. Lohse, Phys. Fluids 6, 611 (1994).

[9] S. Grossmann and D. Lohse, Phys. Rev. E 50, 2784 (1994).

[10] O. Cadot, J. H. Titon, and D. Bonn, J. Fluid Mech. 485, 161 (2003).

[11] S. Ghosal, T. S. Lund, P. Moin, and K. Akselvoll, J. Fluid Mech. 286, 229 (1995).

[12] V. Eswaran and S. B. Pope, Computers Fluids 16, 257 (1988).

[13] S. B. Pope, Turbulent Flow (Cambridge University Press, Cambridge, 2000).

[14] J. Meyers, B. J. Geurts, and M. Baelmans, Phys. Fluids 15, 2740 (2003).

[15] B. J. Geurts, Elements of direct and large-eddy simulation (R.T. Edwards, 2003).

[16] W. van der Water, 2005, private communication. 